\title{
Design Opportunities for Supporting Treatment of People Living with HIV / AIDS in India
}

\author{
Anirudha Joshi ${ }^{1}$, Mandar Rane ${ }^{1}$, Debjani Roy ${ }^{1}$, Shweta Sali ${ }^{1}$, Neha Bharshankar ${ }^{1}$, \\ N. Kumarasamy ${ }^{2}$, Sanjay Pujari ${ }^{3}$, Davidson Solomon ${ }^{4}$, H. Diamond Sharma ${ }^{5}$, \\ D.G. Saple ${ }^{6}$, Romain Rutten ${ }^{7}$, Aakash Ganju ${ }^{7}$, and Joris Van Dam ${ }^{8}$ \\ ${ }^{1}$ Indian Institute of Technology, Bombay \\ ${ }^{2}$ YRG CARE, Chennai \\ ${ }^{3}$ Institute of Immunological Disorders, Pune \\ ${ }^{4}$ SHADOWS, Chirala \\ ${ }^{5}$ Catholic Medical Centre, Imphal \\ ${ }^{6}$ HHRF, Mumbai \\ ${ }^{7}$ Johnson and Johnson \\ ${ }^{8}$ Formerly Johnson and Johnson \\ \{anirudha, mrane\} @iitb.ac.in, \\ \{debjani.r, swetz21, ndm.madame, sanjaypujari, \\ dimsh23, joris.w.vandam\} @gmail.com, kumarasamy@yrgcare.org, \\ drdavidsonsolomon@rediffmail.com, info@hhrfonline.com, \\ \{aganju,rrutten\}@its.jnj.com,
}

\begin{abstract}
We describe a qualitative user study that we conducted with 64 people living with HIV/AIDS (PLHA) in India recruited from private sector clinics. Our aim was to investigate information gaps, problems, and opportunities for design of relevant technology solutions to support HIV treatment. Our methodology included clinic visits, observations, discussion with doctors and counsellors, contextual interviews with PLHA, diary studies, technology tryouts, and home visits. Analysis identified user statements, observations, breakdowns, insights, and design ideas. We consolidated our findings across users with an affinity. We found that despite several efforts, PLHA have limited access to authentic information. Some know facts and procedures, but lack conceptual understanding of HIV. Challenges include low education, no access to technology, lack of socialisation, less time with doctors and counsellors, high power-distance between PLHA and doctors and counsellors, and information overload. Information solutions based on mobile phones can lead to better communication and improve treatment adherence and effectiveness if they are based on the following: repetition, visualisation, organisation, localisation, and personalisation of information, improved socialisation, and complementing current efforts in clinics.
\end{abstract}

Keywords: HIV/AIDS, healthcare, adherence, user study, design for development. 


\section{Introduction}

Adoption of digital technologies, particularly mobile telephony is on the rise in the developing countries. For example, the number of mobile phones in India grew from 52 million in 2005 to 707 million in 2010 [1]. As penetration increases, a large number of new users can potentially get access to the advantages of digital technologies. Among other things, expectations are rising about what technology solutions can do to improve healthcare in these contexts [2-12]. However, as has been acknowledged before [13], [14], these new users, new cultures and new use contexts call for fresh approaches to design of such solutions.

Can new technologies support healthcare in developing countries? If so, what ought to be the nature of such a system and under what constraints will it need to work? We investigate information gaps, problems, and opportunities for design of technology solutions for supporting treatment of people living with HIV /AIDS (PLHA) in India. Our objective is to develop a deep understanding of the ecosystem of a cross section of the PLHA in resource-limited settings and to inform the design of a technology solution that could be effectively used as a healthcare intervention tool, and to probe how such solutions could potentially influence patient behaviours.

\section{Background}

\subsection{About HIV, AIDS, and ART}

The spread of Human Immunodeficiency Virus (HIV) and Acquired ImmunoDeficiency Syndrome (AIDS) has reached epidemic proportions, particularly in developing countries. As of year 2009, an estimated 33.3 million people were infected by HIV, of which 22.5 million live in Sub-Saharan Africa and 4.9 million in Asia. India accounts for 2 to 3.1 million estimated cases [15].

The HIV virus spreads through exchange of infected body fluids, mainly during unprotected sexual intercourse, during blood transfusions, by using an infected syringe needle, or from a mother to her baby. HIV attacks the immune system of the infected person causing a decrease in the number of the CD4+ T helper cells in the blood, resulting in immunologic decline and consequent opportunistic infections (OIs). In the period immediately after the infection, a patient may suffer from some symptoms followed by a clinically latent period, where the patient can remain asymptomatic for several months to years. However, over the long term, the patient's plasma viral load (PVL) increases and a correspondingly his CD4 count drops. When the CD4 count falls below 200 copies per $\mathrm{mm}^{3}$, the patient is especially prone to OIs and is considered to have entered the AIDS stage [16], [17].

As of now, HIV is not curable. Once infected, there is no way to eliminate the virus from the body of the patient. The treatment of HIV consists of antiretroviral therapy (ART), a set of drugs that act at various stages of the life cycle of HIV and work by interrupting the process of viral replication. After initiation of ART, patients may experience adverse symptoms. Some of these occur due to Immune Reconstitution Inflammatory Syndrome (IRIS), a condition where the body begins to resist the infections due to partial immune reconstitution (which is a positive sign, but 
one that a patient could misinterpret). Some symptoms occur due to the side effects of the ART drug. Often, side effects are minor and patients overcome them in time, or with the help of medications. However, in some cases, the symptoms are severe or the ART drug is not effective, and the patient needs a change in the ART regimen [17].

Once a patient is stabilised on ART, his PVL reduces and CD4 count increases gradually, and significant immunological improvement may be seen over the period of one year. Studies have indicated that with $95 \%$ adherence to ART for one year, viral suppression occurs in $80 \%$ patients [18]. In developed countries, the average life expectancy of the PLHA has increased from 10.5 in 1996 to 22.5 in 2005 after the introduction of ART [19]. ART has managed to convert HIV from a "virtual death sentence" to a "chronic manageable disease" [17].

Over a period, the virus develops resistance to a particular ART drug and PVL increases again. Resistance may develop faster in case the adherence is poor or if the PLHA discontinues ART [20]. When this happens, the patient needs another line of ART drugs. Several lines of ART drugs have been developed, but the drugs of the "first line" are usually the cheapest and more widely available [17], [21]. The first regimen also has the best chance for a simple regimen with long-term treatment success [22]. Hence, it is highly desirable that once a patient is initiated and stabilised on ART, he should take his pills regularly, not only for his own sake, but also to avoid the emergence of drug resistant viral strains [23]. PLHA are counselled about potential side effects and the importance of adherence before initiation on ART [17].

\subsection{Prior Studies on Treatment Effectiveness}

Adherence is an important factor in effectiveness of ART and has been a topic of many investigations [18], [20], [23-28]. Costs of ART and financial status of the patient play a major role, particularly in resource-limited settings [25]. But giving away free medication may not be a silver bullet. A study in private clinics in India found that while financial reasons were the ones most commonly cited for breaks in treatment, respondents receiving free drugs had lower adherence [23]. Severe depression, less education, being unemployed, high CD4 count, hospitalisation, side effects, and pill burden were the other factors associated with lower adherence. Reasons for missed doses were "ran out of pills", "travelling away from home", "felt sick or ill", "simply forgot", or "busy with other things". Factors that help in improving adherence include patient's knowledge about side effects, belief towards ART, having developed reminder tools for taking medication, and patient's trust and confidence in the doctor [26]. "Directly observed therapy" is known to have improved adherence and treatment effectiveness [27]. Studies have shown a positive correlation between family support and adherence [28], though family support is usually less in case of PLHA because of the stigma.

The importance of adherence and the need to support the patient in improving adherence has also been researched in domains beyond HIV [29-31]. Klein et al. studied adherence to regimens for four chronic illnesses in natural settings and proposed a model for supporting adherence [31]. They describe an "adherence loop" in which first, the patient needs to believe that he has an illness and develop a mental model of the condition and the therapy. Next, he needs to know what he needs to do, when and how, while strengthening his mental model. Finally, he needs to act, and have the cognitive, physical, emotional, and financial ability to do so. 
There have been several efforts to use technology in HIV treatment, particularly in resource-rich countries. After a meta-review of computer-based interventions for HIV prevention, Noar et al. conclude that such interventions have similar efficacy to human-delivered interventions at lower costs and higher flexibility [11]. Interventions based on mobile phones have also been found to be effective [4], [8], [10]. On a dissenting note, one study shows negative results after the use of a dedicated portable alarm, mainly associated with device failure and confidentiality concerns [32].

Early on, mixed opinions were expressed about the potential of mobile phone interventions in resource-limited settings, mainly due to cost apprehensions [3]. As phone penetration improved, authors have become more optimistic. Two pilot experiments in Uganda and Kenya report that using phones for communication between PLHA, primary health workers, and the clinic staff improved adherence and gave a feeling to the PLHA that "someone cares" [6], [7]. Project Masiluleke in South Africa reports increased calls to the HIV helpline after they sent SMSs to a large number of people [9]. Cell Life uses mobile phones for mobile data collection, and sending messages for HIV prevention, positive living, linkages to clinics, text counselling, and supporting ART in South Africa [12].

There have been studies focussed of parts of the ecosystem of PLHA in developing countries. A survey of PLHA in Kenya found that though 89\% PLHA used phones, only $12 \%$ had ever used it for a healthcare purpose, but $54 \%$ indicated that they were comfortable to receive HIV information on the phone [2]. A qualitative study among PLHA on ART in Peru reports usage of the internet and cell phones [5]. It concludes that information on the internet could be overwhelming, and health interventions based on mobile phones using SMS and voice reminders had many advantages in supporting adherence. (However, this study was not exactly in resource-limited settings. All participants were high school educated and were regular internet users.)

\subsection{The Objective}

While use of technology solutions as healthcare intervention tools have been studied in a variety of settings, sufficient research has not been conducted to understand the settings in which such solutions would be used, and what such solutions should look like in those settings and for those patients to achieve an optimal effect. Particularly, there have been no context-of-use studies that look at the whole ecosystem of patients in resource-limited settings to inform the design of interventions.

The objective of this study is to understand the ecosystem of the PLHA in India, including the financial implications, the operations of HIV clinics, the information needs of PLHA, their pill taking and adherence behaviours, the social, cultural, and economic issues affecting the treatment, and the factors affecting the access and acceptance of technology solutions.

\section{Method}

We started the project with visits to five private-sector HIV clinics across India. In each visit, we did a clinic walk-through followed by fly-on-the-wall observation of the clinic operations. We observed in the waiting rooms, doctors' and counsellors' 
rooms, the pharmacy, and the pathology labs. This was followed by detailed discussions with stakeholders, mainly the doctors and the counsellors.

An ethics committee approval was obtained to interview PLHA. From the five participating clinics, 64 PLHA on ART were recruited. After taking an informed consent, we conducted a semi-structured interview with the PLHA in a language they preferred. Some of the moderators were local HIV counsellors, while others were a part of the design team from a university. Female patients were interviewed in presence of female moderators. The interviews focussed on the following:

- Management of ART regimen: How does the PLHA currently manage his ART regimen? What difficulties does he face doing so in different stages? Has the PLHA devised any techniques to improve his adherence?

- Information needs: How did the PLHA find out information about HIV / ART? What information gaps remained in spite of current efforts? How did the PLHA cope with the large amount of information? Which problems occurred due to lack of information?

- Socio-cultural issues: How did the PLHA deal with social issues such as stigma? What is the role of the ecosystem, i.e. clinics, relatives, friends, NGOs, chemist, family physicians, in treatment and adherence?

- Mobile phone usage: Does the PLHA have access to a mobile phone? What does he use it for? Does he use it in any way to improve adherence? Which opportunities and barriers exist in providing health information on a phone?

Given the sensitivity of the subject and stigma issues, the interviews were conducted in a consulting room at the clinic. If the PLHA consented, interviews were recorded in audio and some relevant artefacts (such as pillboxes, charts, prescriptions, and reports) were photographed. After the interviews, some PLHA agreed to allow the moderators for a home visit, where we observed relevant artefacts. Some PLHA who reported poor adherence were given adherence assistance. For example, some were given seven-day pillboxes. Some PLHA with a high pill burden were helped to draw charts to record their adherence. For PLHA with mobile phones, a Google Calendar SMS reminder was set up at their pill times. Interviews were translated if necessary and analysed using a grounded approach described by [33] to identify the important user statements, observations, insights, breakdowns, and design ideas.

After a gap of 1-2 months, 25 PLHA were re-interviewed. The objective of the second interview was to explore any treatment-related events that may have occurred since the first interview (including symptoms, missed pills, or delays) and to ask follow-up questions resulting from the analysis. The usage and perceptions of the adherence assistance provided to the PLHA at the time of the first interview was also probed. Within 3 months after the first interview, all personal details of the PLHA such as photographs, recordings, and contact information were removed from our records as per the requirements of the ethics committee.

After the fieldwork and analysis, a bottom-up affinity of the findings was built to discover patterns, resolve differences, and identify higher-level insights and design opportunities. These were reviewed in two workshops with doctors, counsellors, NGO representatives, technologists, and designers. 


\subsection{Clinics and the PLHA Profiles}

The clinics and locations in our study were identified in close collaboration with an expert panel of HIV clinicians from across India to ensure representation and diversity of the PLHA population. The locations included two large metros (Mumbai and Chennai, population 10 million+ each), a large city (Pune, population 3.5 million), and two small cities (Imphal and Chirala, population 200,000 each). Our locations covered 4 of the 6 states in India with high prevalence of HIV.

All the clinics in the study are in the private sector, within which they represent different models of operation. Two clinics were consulting offices of senior HIV physicians, their assistant doctors, and some staff. Another was an HIV department in a 405-beded hospital, with 7 HIV specialist doctors and 5 counsellors and a HIV ward with 21 beds. The fourth was a HIV unit in a 150-bedded charitable hospital that provides consultation for a nominal fee of USD 0.33. The fifth was located among a cluster of services that included a private clinic of a senior HIV physician, a private nursing home, a rural primary health centre, and a follow-up facility for government ART PLHA. Each clinic included a pharmacy to disburse ART drugs. A pathology facility was either a part of each clinic or was located near it. Some clinics had professional counsellors, while in others, the doctors did the counselling.

Variability was sought also among the 64 PLHA interviewed. There were 40 men and 24 women. Age varied from 26 to 55 and the average was 37. Education varied from less than primary school (16, including 9 illiterates) to graduate or more (9). Annual family income varied from less than USD 1,333 (19) to more than USD 5,333 (9). Occupations included low-income salaried workers such as drivers and security guards (17), homemakers (17), those with white-collar jobs (9), shopkeepers (8), farmers (6), jobless (4), and daily wages workers (3). A majority (41) were diagnosed HIV positive less than 5 years ago, 16 were diagnosed 5 to 10 years ago, and 7 more than 10 years ago. At the time of the first interview, 18 PLHA had been on ART for less than 3 months, 24 from 3 to 12 months, and 22 for more than 12 months. PLHA were reasonably well-distributed across behavioural variables including levels of adherence, family support, knowledge about HIV and ART, and mobile phone usage.

\section{Findings and Implications to Design}

\subsection{Clinic Operations}

\section{Visiting Clinics is a Burden on PLHA's Time}

We found opportunities to speed up clinic operations and minimise the PLHA waiting time with the help of an information system. HIV clinics are crowded places. One reason for this is that good HIV clinics are few. The PLHA spend a long time in the waiting room. A consultation may turn out to be unpredictably long. At times, the doctor may determine that he requires additional diagnostics, before he can make a decision. The counsellor may not be sure whether the PLHA knows enough, so she may repeat information that the PLHA already knows. A doctor remarked, "Our appointment system breaks down every day". 
Secondly, some PLHA consider a tertiary HIV clinic as a place of primary care. For example, a PLHA travelled $200 \mathrm{~km}$ to a HIV clinic because of a minor fever. All he was prescribed was a paracetamol, which he could have easily procured near his home. Could a phone-based system be used to help the patient decide whether they should go to the specialty clinic, or just to a local pharmacy or a family doctor? We found that the clinics are willing and much interested to provide such phone consultations for stable PLHA with known history and minor complaints, but need technology support to do so. The time that PLHA spend at a clinic may discourage follow up. Some PLHA stable on ART have been purchasing pills from the pharmacy, but have skipped meeting the doctor for a long time.

Solutions also need to be culturally grounded. Clinics usually do not pro-actively follow up with PLHA. This seems to be a cultural practice in the medical profession in the private sector. The doctor may suggest a date during consultation, but it is up to the PLHA to determine the actual date of his next visit. Asymptomatic PLHA tend to postpone their clinic visits. For example, a PLHA gave his blood for a CD4 test on doctor's advice, but did not turn up to collect the reports and follow up for 3 months.

All clinics were equipped with computers, but only a few had systems for managing clinic visits. Information systems could speed up clinic operations and implement best practices across clinics (e.g. trigger automated follow-up reminders to PLHA for clinic visits) within the current cultural norms.

\section{Poorly Maintained Health Records}

Technology solutions can help digitise the reports and the prescriptions securely and make them available to doctors across clinics on demand. Many PLHA (particularly the educated ones) meticulously maintain their reports and prescriptions. On the other hand, some PLHA deliberately burn or tear off their papers due to stigma and disclosure issues. Others retain only the latest prescription. At times, some PLHA forget to bring their papers to the clinic and the doctors have no reference to treatment history.

Some clinics maintain a digital or paper copy of the prescriptions and reports, but this leads to additional data entry, data duplication (and possible inconsistency), and slowing down of the clinic operation. In some cases PLHA hop clinics, because of treatment failures, financial constraints, or seasonal migration. Unfortunately, there are no arrangements for the PLHA to move their records digitally between clinics.

A related issue is the language of the reports and the prescriptions. Records are primarily useful for the pathologists, the doctors, and the pharmacists as they are in English, and are often cryptic. Many PLHA do not read English. If they were to maintain their records, these would be of little use to them personally.

Digital records would not only make data available at all times, but also speed up the clinic operation and improve communication with patients. Versions of the reports and prescriptions can be translated, elaborated and simplified in a language that PLHA understand, and made available through a medium that they can use.

\subsection{Financial Issues}

Technology solutions need to operate under tight financial constraints, but also can empower the PLHA with prudent financial planning. The cost of ART varies from 
very expensive to almost free depending on where the PLHA gets his treatment. In the private sector, the current cost of the drugs could vary from USD 266 to USD 5,866 per year. Additional expenses are required for diagnostics (CD4 tests, viral load tests, resistance tests, etc.) and the doctors' consultation. These can add up to a substantial percentage of the annual income for many. (India's average nominal per-capita annual income is about USD 1,176 [34].) On the other hand, treatment is available for free in government hospitals since 2004 [17]. (It must be pointed out that the PLHA in this study were recruited in private clinics. PLHA taking treatment exclusively at government hospitals were not represented. They might have different perceptions.)

As discussed above, free treatment may not be the solution, as free treatment was found to be correlated with poor adherence [23]. One reason for this could be nonawareness of treatment costs. Our sample did include PLHA who got free treatment. Among these, some PLHA took consultation (or second opinions) from a private physician, but got their ART drugs and tests done from a government hospital. Others were associated with clinical trials at the private clinics and were entitled to free medication. Many of these PLHA were not aware of the cost of their medication. Further, even the PLHA paying for their treatment were not aware of the cost of the next line of treatment, which they would have had to take had they failed their current regimen. Technology solutions should make costs of free ART transparent.

Some PLHA perceived that more expensive medicines were "serious" or good, and given a choice, chose these if they thought they could afford them. Doctors and counsellors pointed out that some people may agree to expensive arrangements to begin with, but cannot keep up as the expenses mount.

Costs other than the treatment are also significant. PLHA need to make at least 4 trips to the clinic every year [17]. For each trip, a PLHA has to bear the cost of travel and the loss of wages of half to two days. Due to the stigma, some PLHA prefer to travel to a clinic further from their residence, with the hope of retaining anonymity. Of the 64 PLHA, 24 travelled for more than 1 hour each way to reach the clinic, of which 13 travelled for 4-8 hours each way. Further, PLHA on ART need to take good nutrition and improve their lifestyle. Unfortunately, these changes are beyond the means of some. When asked whether he drinks boiled water as advised by the counsellor, a PLHA from lower-middle class found the idea laughable - it was way beyond his means to do so.

Unfortunately, some people are not able to foresee and plan their expenses for a lifelong ART. For example, a farmer on ART with very limited means on his second trip to the HIV clinic had had to sell a cow to meet expenses of each of his trips. He had another 4-5 cows, but did not have a plan of what would happen after he sold them all. When probed, all he could say was "we shall see when the time comes".

Technology solutions could help PLHA plan their finances better, but they need to operate under existing constraints. Adding devices, special mobile phones, or services may not be scalable, as resources are already scarce. Giving away devices may not work even if funding could be found, partly because of the possibility of a stigma on these devices, and partly because it would add another disruption to the life of the PLHA. Solutions could instead try to leverage existing infrastructure (such as ordinary mobile phones) or shared resources (such as waiting rooms in clinics). 


\subsection{Awareness Issues and Information Gaps}

\section{Insufficient Counselling}

Interactive learning tools could complement, support and personalise current counselling efforts. Counselling the PLHA is important on diagnosis and before initiation on ART. Clinics do counsel on both the occasions, but counselling is difficult on these occasions as the PLHA is in a state of shock and is not in a condition to absorb information. Secondly, the field of HIV has too much information, not all of which may be relevant to an individual. Thirdly, counselling a large number of PLHA is tedious, and after a while, the counsellor could get fatigued.

Doctors and counsellors spend a lot of effort repeating similar information to each PLHA. However, an individual PLHA gets very little time with the doctor or counsellor to absorb information and ask questions and doubts. There is always a long queue and time pressure. Further, counselling once is not enough, particularly for less educated and older PLHA who require a lot of repetition and redundancy. Group counselling (as practiced in some government clinics) can reduce the load to some extent. Nevertheless, it is less effective than individual counselling as PLHA do not speak up or ask doubts in a group.

It was observed that HIV brochures and other material in the waiting rooms of the clinics were often left untouched by PLHA. This could be partly because of low levels of literacy, but partly because the brochures are not designed to address the needs of the individuals. We came across a government ART centre that recently started screening videos related to HIV and ART in the waiting room. This seemed more effective and it was observed that the PLHA were more attentive. Most clinics, however, screen TV channels for entertainment in their waiting areas. Informational videos and other forms of self-paced learning material could be a good tool to prime the PLHA before the counselling and repeat the information after the counselling. These would optimise the time that the PLHA spend in the waiting rooms, and leave more time for personalised discussion during the counselling session. Tool-use logs could give hints to counsellors on where they need to focus.

Bombarding information in one direction may not be good enough. It was observed that asking PLHA probing questions about their knowledge of HIV during the interviews triggered their curiosity and encouraged them to ask questions to the moderators. We suggest that the learning tools could quiz the PLHA about their knowledge. This would not only help evaluate the effectiveness of the counselling and identify weak areas, it would also prime the PLHA to absorb more information.

Information needs to be localised beyond language. PLHA use different terminologies to describe the same symptom. Food, meal timings, and lifestyle vary between states, and because of professions. For example, in Imphal, most people have lunch at 8 am and dinner at $8 \mathrm{pm}$, and no other meals in between. Nutritional advice and pill times will need to be adjusted accordingly.

Technology could be used to repeat counselling, to personalise it according to literacy levels and medical conditions, to localise it in terms of language, food, profession and culture, and to make it available on-demand and in a form that people, including those with less education, can follow. A technology-based solution could also ask questions, give feedback based on responses, and evaluate and track the 
effectiveness of the communication. As we discuss below, it could also help PLHA connect with other experienced PLHA anonymously to get their questions answered.

\section{Unfamiliar Terminology}

Solution designers need to consider that some PLHA may not be aware of relevant terminology. It was found that some clinics referred to the ART drugs they prescribed simply as "HIV medication", perhaps with the good intention of avoiding jargon and simplifying communication. As a result, though, some of the PLHA on ART had never heard of the term ART. Others had heard the term ART, but they associated it with the free medication available in government hospitals, or with the ART centre in government hospitals. Such a PLHA is likely to ignore all communications about "ART", as he would consider these irrelevant.

Similarly, many PLHA were not familiar with relevant terms such as CD4, viral load, or the virus. Not knowing common terms or not understanding their precise meaning prevents PLHA from communicating with each other (as we discuss below).

\section{Issues with Pill-Taking and Adherence}

Solutions are needed to help PLHA take their pills and improve adherence on a dayto-day basis. It was observed that the pharmacists often explain orally to PLHA how to take their pills while handing them over. They tend to do this if the PLHA is newly on ART or if he asks for an explanation. Yet, a few PLHA were not completely aware of how they should take their drugs. For example, one woman did not know that her daughter's pills were dispersible, and had a difficult time in making her swallow them. Another woman was not aware that her pill was supposed to be taken just before going to bed - she complained of giddiness, a side effect. Another woman cut her pill into two and had it twice a day (a wrong practice), because she had difficulty in swallowing it whole. Patients with complex regimens (with 5+ pills per day) needed visual support (such as a chart or timings written on pill bottles), especially in the first few weeks.

Many of the less educated PLHA could not recall the names of their drugs. They recognised and differentiated between their pills visually - mainly by colour, shape, bottle, or a few alphabets on the label. Confusions did arise if a company changed its pill design, or when the pharmacy provided a different brand of the same drug.

In a system meant to support PLHA in pill taking, reading out drug names from the prescription may not be enough. Additional cues such as a photograph or shape and colour of the pill should be communicated. It should be accompanied by pill-taking instructions, health tips, and answers to frequently asked questions.

Many PLHA reported having missed a few pills, but pill counts revealed that they often underestimated the missed pills. It was found that many PLHA faced difficulty in counting days since last visit to the clinic, accounting for the number of missed pills, and then estimating the number of pills left. One of the PLHA who found this task difficult was a carpenter, who was otherwise able to make complex calculations routinely. It was observed that some older, less educated PLHA faced difficulties in reporting their age or in counting cash. A system should work within these constraints and provide personalised data and feedback about pill counts and estimates.

People for whom the SMS alarms were set up were happy to get their SMS beeps (though some never read the SMS - "no one I know would ever send me an SMS"). 
These PLHA were disappointed when we informed them that their alarms had to be discontinued after 3 months of the first interview (as per the ethics committee requirement). These personalised pill reminders had clearly helped the PLHA and were not considered intrusive. Even those who were already using mobile phone alarms were happy to receive SMS reminders, as the redundancy ensured that the system was fail-safe.

\subsection{Knowing Facts and Procedures or Understanding Concepts?}

Solutions need to help the PLHA build a mental model of HIV and ART apart from informing about facts and procedures. In spite of several awareness issues and information gaps, some factual and procedural knowledge about HIV and ART is slowly getting built up among PLHA. However, many have a poor conceptual understanding of HIV and ART. For example, some PLHA know that ART is the treatment for HIV (a fact), and that they should take their pills regularly (a procedure), but cannot explain why they need to take their pills lifelong, or what would happen if they missed their pills or took them late. As Klein et al. suggest [31], unless the PLHA develop a mental model, they would not believe in the therapy and would find it difficult to understand what to do, particularly in a new situation.

\section{HIV - "Everybody knows, but nobody understands"}

Through the awareness campaigns in several media, many have heard of HIV. However, few understand how HIV actually works. When asked what HIV was, the first reaction of a PLHA was "everybody knows what HIV is". When the moderator insisted that he explain to him as if he was a newly infected, naive person, he quickly replied that he "did not know that much". People usually think about a disease in terms of its symptoms. They try to describe HIV in terms of darkening of the face, weight loss, body ache, death, etc. Unfortunately, in case of HIV, PLHA may face different symptoms, so they face difficulty in coming to grips with this disease.

Most PLHA (though not all) had reasonable procedural knowledge. They knew about the modes of transmission of HIV (unsafe sex, injection needles, razors, blood transfusion, and mother to child transmission). However, they could not explain why HIV spreads through these modes and not through other forms of contact.

Many PLHA betrayed their conceptual confusions (or possibly denial). One PLHA thought he was infected because he "donated blood". A PLHA responded, "I don't know how the virus came in our house - we always keep it clean". Another wanted to know from the moderator whether she could cook for her family or serve food. Some had become overcautious about hygiene and avoided touching children, shaving in saloons, sharing utensils, or having sex.

\section{CD4 - "Batting Without Knowing the Score"}

Many PLHA had heard about a CD4 count, though some had gotten it mixed up with viral load. Some remembered the numbers ( "87"), but were not sure if more CD4 was good or bad, or what a good value should be. Others were not sure what CD4 was and why they need to be tested for it so regularly. To draw an analogy with cricket, these people are batting without knowing the score. 
Since it is usually not possible for clinics to proactively follow-up PLHA, the onus of going to the HIV clinic regularly is on the PLHA. If the PLHA is non-symptomatic and not yet on ART, he may postpone visiting the clinic though his CD4 count could be dropping. None of the PLHA was aware that the CD4 count has a possibility of $20 \%$ error and PLHA with counts below 250 should test frequently [17].

The duration between CD4 tests for many was longer than the prescribed 3-6 months. Many had OIs at the time of initiation of ART. To continue with the cricket analogy, these batsmen started trying to catch up on their run-rate after the asking-rate had gotten out of hand.

\section{ART, Adherence, "Scolding", and "Side Effects"}

Many PLHA had factual and procedural knowledge about ART. They knew that ART "improves health". They also knew that they have been told to be adherent to their pills and to take them on time. However, when we asked why they should be adherent, a frequent answer was, "otherwise the doctor will scold me".

Only procedural and factual knowledge is not enough when one has to handle a situation that one has not been prepared for. Many reasons for non-adherence seem to be related to a poor understanding of ART. For example, half an hour after the time for the dose, a PLHA remembered that he had not taken his pill for the day, so he skipped his dose. According to the doctors, an occasional half-hour delay would not have mattered. Another PLHA has been counselled that alcohol consumption has bad effects on ART. Therefore, on an evening when he plans to consume alcohol, he deliberately skips his ART pill. Another PLHA waits for the exact time to take his pill. His pill time is 8:30. He sets an alarm for 8:28, and when the alarm rings, he takes a pill out of the bottle and waits for the next two minutes before he takes it. Some PLHA were not sure whether they should take a double dose the next time if they miss a pill, or whether they should delay the current dose if the last dose was late. Some were unsure if they could share pills with their spouse. These situations could be avoided by developing a mental model of HIV and ART.

In the period immediately after initiating ART, PLHA may experience symptoms and an apparent deterioration of health. A symptom could be a side effect, an OI, an IRIS condition, or an indication of a regimen failure. Each of these calls for a different medical follow-up. Most PLHA could not differentiate between these. They called each of these a "side effect" of ART. One PLHA believed that his wife and child died "because of ART" and was afraid that he was now put on the same medication. Poor conceptual understanding of ART could lead to self-treatment, discontinuation of ART, or the PLHA being lost to follow-up.

Even in cases where the ART is successful and the health improves, conceptual understanding of ART is important. Some PLHA did not know that ART was meant to be taken for the rest of the life. After feeling better, they had felt that they had recovered and had discontinued ART. Others are "bored" of taking tablets daily. Unfortunately, missing a pill has no immediate visible effect that could help the PLHA build a mental model of how ART works. As a PLHA poignantly put it, "it makes no difference, whether I take the pill, or I skip it”. Missing a dose helps the virus build resistance and this leads to a treatment failure in the long term. Technology solutions can help externalise these abstract notions, and provide continuous feedback and encouragement for adherence and health status. 


\section{Conceptual Clarity Leads to a "Normal" Life}

Some PLHA did have a good conceptual understanding of HIV and ART and could explain it in their words. One PLHA remarked, "HIV is a virus, not a disease". Another explained CD4 were the "germs of power" and OIs as "your power goes down and all diseases catch you”. Another explained that when ART is successful, "the virus becomes sleepy". Most of these PLHA also demonstrated a positive approach to life and were adherent to their treatment.

Experience of suffering coupled with conceptual understanding of the disease seemed to make people compliant with the treatment. PLHA who suffered a lot before feeling better with ART, and those who have seen others die of HIV seemed to have a better adherence. Technology solutions can communicate positive and negative experiences of other PLHA to help build a mental model and minimise the personal suffering of the individual.

\subsection{Social and Emotional Aspects}

\section{Family Support - The Haves and the Have-Nots}

Solutions need to be personalised, depending on the disclosure status of the individual within his family. Some of the PLHA who had disclosed to their families got both financial and emotional support. Such support is particularly valuable at the initiation of ART, when the pill burden is high and the regimen is complex.

Many PLHA had disclosed not to the entire family, but to one or two members. Mature, open minded, supportive, and earning members of the family were preferred for disclosure. In many cases, PLHA preferred a same-gender disclosure, in other cases, disclosing to males was preferred, mainly due to the financial implications. The people who were often kept in the dark were the elderly ("why bother them") and the children ("they might tell the neighbours").

Full disclosure is not necessary to gain support. Family members (especially children) are happy to support even without a full disclosure. Many PLHA had only disclosed the need for taking regular pills, while others had said that they suffer from a non-stigmatising chronic illness such as blood pressure or diabetes.

Though desirable from a treatment perspective, many PLHA struggled to disclose their status to family members. In some cases, disclosure led to tension in the family, break up of a joint family, or separation from spouse. Due to such fears, a few PLHA have preferred to disclose to friends, but not to family members. Such PLHA tend to reject a solution that has a risk of accidental disclosure to the family. For example, one PLHA who also had tuberculosis preferred treatment in a private clinic because government clinics insist on DOTS (directly observed treatment, short course) - he did not wish to let anyone visit his home to observe him taking pills.

Non-disclosure to family members creates obstacles for ART. A PLHA avoided using alarms because his family members might get suspicious and ask why he was setting an alarm for odd hours such as 9 am or $9 \mathrm{pm}$. Another PLHA took his pills at night, when everyone had gone off to sleep. Some tore off labels of pill bottles and hid them in a safe place. A PLHA complained of the feeling of being "alone", because of such situations.

A system meant to support ART has to be flexible. For a PLHA who enjoys high levels of family support, such a system should empower family members and help 
them give better support. A PLHA who has not disclosed to family members needs support all the more, but such support needs to be more discreet and under the control of the PLHA. Similarly, a PLHA with partial disclosure would need other solutions. In no situation should such a system cause unintended disclosure, as it would have disastrous consequences for the PLHA, and it would compromise privacy laws.

\section{Need for Anonymous Socialisation}

Technology-mediated social networking has become common. We found that there is both a need and an opportunity to leverage technology to enable socialisation among PLHA, although in a new form.

Socialisation is the usual way for people to acquire information in India. Even while looking up an address, Indians prefer to ask someone on the street for directions rather than look up a map or a signage. However, we found that PLHA socialise less. To begin with, some PLHA had difficulty in locating a good HIV clinic. Being shy about asking someone and not knowing where to look does not help. Some PLHA avoid social functions to maintain ART times. Others avoid socialisation because of stigma, depression, or because of the need to save money, as socialisation often leads to expenses. While PLHA socialise less, on the other hand, lack of socialisation gives PLHA a feeling of being "all alone".

We found that PLHA do have a lot to share. Some PLHA use interesting ideas for improving ART adherence or to integrate it in their lives. A PLHA on two ART pills a day uses an interesting "two-pill strategy" to remember whether he has taken his current dose (a common problem). He takes his ART pills from a small pillbox. Every night, after he has taken his pill, he takes out two pills from the bigger bottle and keeps them in the pillbox. If the pillbox has one pill at night, it implies he has not taken his night pill yet, but if it has two or zero pills it implies that he has taken it. Conversely, if it has two pills in the morning, it implies that he not taken his morning pill yet, but if it has only one pill, then it implies that he has taken it. Another PLHA with a travelling job ensures that he never forgets to carry his pills - he always carries three things, "phone, money, and ART". Another PLHA thought of his ART as his religion. Some PLHA associate taking a pill with events in a day - sending children to school, or a meal. Each of these ideas seems to be worth spreading.

Informal social activities among PLHA seemed to be happening in some contexts. In a rural hospital, PLHA were aware of each other's problems and there was exchange of information among them. It appeared that the hospital gave the PLHA anonymity and the knowledge that others are PLHA like them. In an urban government hospital, some ART-experienced PLHA were invited to share tips with others after a group counselling session. A PLHA who attended it reported that he valued such tips more than what the counsellor had told. Unfortunately, such socialisation among the PLHA was not observed in the urban private clinics.

In some cities, formal networks of positive people and support groups exist outside the clinics. The activities of these networks seem to be growing and their members benefiting. However, only a few of the PLHA in our study were active in positive networks, hinting that these are not yet widespread. Positive networks may not be suitable for those who live in rural areas or for those who have travelling jobs. Others may not prefer them because of stigma and face-to-face nature of their activities. 
Typically, online social networking forums are meant to reach out to existing and new friends with one's true credentials. Such social networking might not work for PLHA due to stigma issues. However, anonymous networks, possibly on mobile phones, and catalysed by the clinics could help PLHA socialise.

\section{Power Distance, Trust, and Fear}

India is considered a society with a high power distance index with a rank of 17 out of 68 countries and regions [35]. In a high power distance society the less powerful people accept and expect that power is distributed unequally. While characterising a country along a few dimensions thus could be critiqued as not being the best way to understand the rich textures of its society, high power distance is certainly visible between the PLHA and the doctors, and to an extent the counsellors. Not only did PLHA respect and trust the doctors, they seemed to revere them. A doctor mentioned that he observed some PLHA leave his clinic walking backwards, something that they would only do in a temple. A doctor showing quick results is particularly revered. No other source of information, including TV, radio, internet, a positive network, or a friend is trusted as much as the treating doctor.

Unfortunately, the high power distance also prevents communication. Many PLHA are afraid to ask too many questions to the doctor, as it might seem that they are questioning the doctor's judgement. Gender gap too plays a similar role. Most HIV doctors in our study are men, and women are shy to ask them simple questions such as "can I cook for my family?" (A PLHA woman asked this question to the female moderator). Conversely, men are shy to talk with counsellors (who happen to be mostly women) about multiple sex partners. Time pressure in the clinic makes it harder to overcome the power distance.

As has been said earlier [13], technology could possibly bridge some of these social gaps. It could provide a platform to the PLHA to ask their questions offline (or to just listen to others' questions) and get the doctor's answer. At the same time, the trust with the doctor could be leveraged by using the doctor's voice and / or his photograph while giving medical advice.

\subsection{Non-uniform Mobile Phone Usage}

Of particular interest to us were the differences in mobile phone usage patterns among PLHA. Though penetration of mobile phones is quite high, mobile usage abilities of the PLHA varied a lot. Of the 64 PLHA interviewed, 42 had access to a personal or a shared mobile phone. Almost all of them could receive and make calls. Only 33 could look up missed calls, and 30 could look up a number in the phone book. Only 20 had ever read an SMS, and 9 had ever written one. Only 17 reported using noncommunication functions of the phone such as the alarm, the radio, or the camera. This being a qualitative user study, the sample might not represent the population proportionately, but it certainly indicates the constraints that a technology solution would encounter.

Even though a facility is available, not everyone might think of using it for ART. Many PLHA with mobile phones had never thought of using an alarm as a pill reminder. By the second interview, some had started using alarms in their mobile phones (possibly because of our first interview). Similarly, people who were 
presented with a seven-day pillbox - a relatively simple intervention - said that they found it useful ("we can tell if we have missed a dose") and reported that their adherence had improved. Once PLHA start using it, an intervention may prove to be useful. However, many PLHA may not initiate the use by themselves. The intervention has to be initiated at the clinic along with ART and a PLHA has to be walked through the first few steps.

Phone sharing is another area that needs consideration. A phone is frequently shared between family members. In some cases, a child is more tech-savvy than its parents. For example, a PLHA said that his daughter could look up an SMS, while he could not. Even so, he did not mind receiving HIV related SMS. He said, "You send, madam. I will manage". On the other hand, a tech-savvy PLHA preferred emails "because these are more secure" than SMS "which could be read by anyone".

\section{Conclusions and Ongoing Work}

The problem of treatment of people living with HIV / AIDS in resource-limited settings such as India has moved, at least in part, from the medical and pharmaceutical domain to the information and communication domain. Many issues relate to low education, access to technology, access, presentation and interpretation of information, behavioural aspects, lack of socialisation, less time with doctors and counsellors, high power distance between PLHA and doctors and counsellors, and information overload. This study establishes that there is a clear need and an opportunity to make technology-based, user-centred interventions in management of anti-retroviral therapy. Technology presents opportunities, but realising them may not be easy, given the usability problems and information-seeking behaviours. A specific, isolated intervention (such as only SMS reminders) could have a limited effect, or could even be counterproductive.

This study points to the need for a holistic intervention. Gains can be achieved by considering not only the medical needs of the patient, but also the informational, social, and emotional aspects, and the abilities of the people. Given the variability across the PLHA, the system should have flexibility. The intervention should be personalised for the needs of an individual, based on education, knowledge, availability of family support, medical status, age, gender, and treatment. It should be localised for a community, based on language, food habits, profession, and culture. Many PLHA will need repetition of information as much of it is new to them; most will need help to build a conceptual understanding of HIV and ART.

The intervention should be grounded in the reality of a resource-limited setting. It should not add to the already heavy financial burden. It should not be a stand-alone, independent activity, but and should closely complement the ongoing efforts in the clinic. Trust can be leveraged and simultaneously barriers of power distance can be broken with a usable interface between the doctor and the patient. On the other side, the system can improve the efficiency of the clinic, secure health records, and optimise the time of the PLHA in the clinic.

The last decade has brought digital technologies within the reach of new users from developing economies for the first time. This study brings insights about the needs and contexts of these users. Though our focus was on HIV and ART, our study 
resulted in several broad-based findings as well. These can be potentially applied to supporting other chronic illnesses and conditions with technology solutions.

Based on the insights from this study, we have undertaken design of an interactive voice response system on the PLHA side, and a web-based system on the clinic side. Our next step would be to prototype such a system, evaluate it for usability, and perform a randomised control study among a cohort of PLHA to evaluate its effectiveness on ART adherence and pathological outcomes.

Acknowledgements. This project was funded through a grant from Johnson \& Johnson Limited. We thank the doctors, counsellors, moderators, and the people living with HIV / AIDS for participating in this project and sharing their insights.

\section{References}

1. TRAI: Telecom Subscription Data as on (October 31, 2010), http://www.trai.gov.in/WriteReadData/trai/upload/ PressReleases / 780 / PRecodiv24dec10 .pdf (accessed December 24, 2010)

2. Lester, R., Gelmon, L., Plummer, F.: Cell phones: tightening the communication gap in resource-limited antiretroviral programmes? (2006)

3. Kaplan, W.: Can the ubiquitous power of mobile phones be used to improve health outcomes in developing countries? (2006)

4. Vidrine, D., Arduino, R., Lazev, A., Gritz, E.: A randomized trial of a proactive cellular telephone intervention for smokers living with HIV/AIDS (2006)

5. Curioso, W., Kurth, A.: Access, use and perceptions regarding Internet, cell phones and PDAs as a means for health promotion for people living with HIV in Peru (2007)

6. Chang, L., Kagaayi, J., Nakigozi, G., Packer, A., Serwadda, D., Quinn, T., Gray, R., Bollinger, R., Reynolds, S.: Responding to the Human Resource Crisis: Peer Health Workers, Mobile Phones, and HIV Care in Rakai, Uganda (2008)

7. Lester, R., Karanja, S.: Mobile phones: exceptional tools for HIV/AIDS, health, and crisis management (2008)

8. Kevin Patrick, K., Griswold, W., Raab, F., Intille, S.: Health and the Mobile Phone (2008)

9. Fabricant, R.: Project Masiluleke. Interactions 16(6) (2009)

10. Winchester, W.: Catalyzing a Perfect Storm: Mobile Phone-Based HIV-Prevention Behavioral Interventions. Interactions 16(6) (2009)

11. Noar, S., Black, H., Pierce, L.: Efficacy of computer technology-based HIV prevention interventions: a meta-analysis (2008)

12. Benjamin, P.: Cellphones 4 HIV. In: mHealth Potential in South Africa: The Experience of Cell-Life (2010), http: / / www . cell-life.org/images / downloads / CellLife_Organisation_Poster.pdf (accessed 2010)

13. De Angeli, A., Athavankar, U., Joshi, A., Coventry, L., Johnson, G.: Introducing ATMs in India: a contextual inquiry (2004)

14. Joshi, A.: Mobile Phones and Economic Sustainability - Perspectives from India. In: Workshop on Expressive Interactions for Sustainability and Empowerment, Londan (2009)

15. AVERT: Worldwide HIV \& AIDS Statistics Commentry, http: / /www. avert.org/worlstatinfo.htm (accessed 2009)

16. AVERT: AIDS, http://www. avert.org/aids.htm (accessed 2009) 
17. NACO: Antiretroviral Therapy Guidelines for HIV-Infected Adults and Adolescents Including Post-exposure Prophylaxis. Antiretroviral Therapy Guidelines for HIV-Infected Adults and Adolescents Including Post-exposure.pdf,

http: / / nacoonline.org/upload/Policies\&Guidelines / 1

(accessed May 2007)

18. Arnsten, J., Demas, P., Gourevitch, M., Buono, D., Farzadegan, H., Schoenbaum, E.: Adherence and Viral Load in HIV-infected Drug Users: Comparison of Self-report and Medication Event Monitors. In: Conference on Retroviruses and Opportunistic Infections, New York (2000)

19. Harrison, K., Song, R., Zhang, X.: Life Expectancy After HIV Diagnosis Based on National HIV Surveillance Data From 25 States, United States (2010)

20. Sherr, L.: Understanding Adherence (2000)

21. Bartlett, J., Shao, J.: Successes, challenges, and limitations of current antiretroviral therapy in low-income and middle-income countries (2009)

22. Panel on Antiretroviral Guidelines for Adults and Adolescents: Guidelines for the Use of Antiretroviral Agents in HIV-1-Infected Adults and Adolescents,

http: //www.aidsinfo.nih.gov/contentfiles/ Adul tandAdolescentGL.pdf (accessed January 10, 2011)

23. Sarna, A., Pujari, S., Sengar, A.K., Garg, R., Gupta, I., Dam, J.: Adherence to Antiretroviral Therapy \& its Determinants Amongst HIV Patients in India (2008)

24. Nischal, K., Khopkar, U., Saple, D.: Improving Adherence to Antiretroviral Therapy (2005)

25. Duraisamy, P., Ganesh, A., Homan, R., Kumarasamy, N., Castle, C., Sripriya, P., Mahendra, V., Solomon, S.: Costs and Financial Burden of Care and Support Services to PLHA and Households in South India (2006)

26. Wu, X.: Factors associated with adherence to antiretroviral therapy among HIV/AIDS patients in rural China (2007)

27. Goggin, K., Liston, R., Mitty, J.: Modified directly observed therapy for antiretroviral therapy: a primer from the field (2007)

28. Cauldbeck, M., O’Connor, C., O’Connor, M.B., Saunders, J. A., Rao, B., Mallesh, V., Kotehalappa, N., Kumar, P., Mamtha, G., McGoldrick, C., Laing, R.B., Satish, K.S.: Adherence to anti-retroviral therapy among HIV patients in Bangalore, India (2009)

29. Osterberg, L., Blaschke, T.: Adherence to medication, pp. 487-497 (2005)

30. PhRMA: Just What the Doctor Ordered: Taking Medicines as Prescribed Can Improve Health and Lower Costs,

http: / / www.phrma.org/files/attachments / Adherence.pdf (accessed March 2009)

31. Klein, D., Wustrack, G., Schwartz, A.: Medication adherence: Many conditions, a common problem. In: 50th Annual Meeting on Human Factors and Ergonomics Society (2006)

32. Mannheimer, S., Morse, E., Matts, J., Andrews, L., Child, C., Schmetter, B., Friedland, G.: Sustained benefit from a long-term antiretroviral adherence intervention (2006)

33. Beyer, H., Holtzblatt, K.: Contextual Design. Morgan Kaufmann, San Francisco (1998)

34. IMF: Report for Selected Countries and Subjects,

http://en.wikipedia.org/wiki/

List_of_countries_by_GDP_nominal_per_capita (accessed October 2010)

35. Hofstede, G.: Geert Hofstede Cultural Dimensions, http: //www.geert-hofstede.com/hofstede_dimensions.php (accessed 2009) 